Check for updates

Cite this: Mater. Horiz., 2020,

7, 2085

Received 19th January 2020,

Accepted 13th May 2020

DOI: $10.1039 / \mathrm{d} 0 \mathrm{mh} 00100 \mathrm{~g}$

rsc.li/materials-horizons

\section{Self-powered integrated system of a strain sensor and flexible all-solid-state supercapacitor by using a high performance ionic organohydrogel $\dagger$}

\author{
Jianren Huang, (D) $\ddagger^{\mathrm{abc}}$ Shuijiao Peng, $\ddagger^{\mathrm{a}}$ Jianfeng Gu, $\ddagger^{\mathrm{a}}$ Guoqi Chen, ${ }^{2}$ \\ Jianhong Gao, ${ }^{c}$ Jin Zhang, ${ }^{a}$ Linxi Hou, (D) ${ }^{a}$ Xiaoxiang Yang, ${ }^{c}$ Xiancai Jiang (D) *a and \\ Lunhui Guan (D) *b
}

The rapid development of human-machine interface technologies is dependent on flexible and wearable soft devices such as sensors and energy storage systems. One of the key factors for these devices is the hydrogel electrolyte. In this work, firstly we constructed an anti-freezing, self-healing, adhesive and tough conductive double network ionic organohydrogel by simultaneously introducing $\mathrm{NaCl}$ and glycerol (Gly) into poly(vinyl alcohol)/poly(acrylic amide-acrylic acid) (PVA/PAMAA). Then, based on this organohydrogel, a strain sensor and a flexible all-solid-state supercapacitor were assembled separately and their properties were measured. The fabricated strain sensor showed a highly sensitive response (gauge factor $=8.303$ ) in a broad strain range (from 500 to $1000 \%$ ), enabling the accurate and reliable detection of various mechanical deformations. Meanwhile, the flexible all-solid-state supercapacitor demonstrated a specific capacitance of $75.75 \mathrm{mF} \mathrm{cm}{ }^{-2}$, high cycling stability $(90.2 \%$ retention rate after 5000 cycles) and excellent mechanical stability. Lastly, a self-powered integrated system composed of the strain sensor and flexible all-solid-state supercapacitor was fabricated. The sensor can be driven by the supercapacitor and operate stably in a wide strain range sensing test with low power consumption. More importantly, the self-powered integrated system could be directly attached to the human body to detect human motions, showing its great potential application in wearable devices. This work would enlarge the research and application of high-performance ionic hydrogel electrolytes.

\footnotetext{
${ }^{a}$ College of Chemical Engineering, Fuzhou University, Fuzhou 350108, China. E-mail: jiangxc@fzu.edu.cn

${ }^{b}$ CAS Key Laboratory of Design and Assembly of Functional Nanostructures, Fujian Key Laboratory of Nanomaterials, Fujian Institute of Research on the Structure of Matter, Chinese Academy of Sciences, Fuzhou 350108, China.

E-mail: guanlh@fjirsm.ac.cn

${ }^{c}$ School of Mechanical Engineering and Automation, Fuzhou University, Fuzhou 350108, China

$\dagger$ Electronic supplementary information (ESI) available. See DOI: 10.1039/d0mh00100g

\$ Authors with equal contributions.
}

\begin{abstract}
New concepts
Stretchable sensors for humans have attracted the attention of many researchers. However, conventional sensors have to be driven by external energy supplies. In this paper, an integrated self-powered integrated system composed of a sensor and a flexible all-solid-state supercapacitor was fabricated based on a poly(vinyl alcohol)/poly(acrylic amide-acrylic acid)/ glycerol/NaCl (PVA/PAMAA/Gly/NaCl) double network ionic organohydrogel. Firstly, PVA/PAMAA/Gly/NaCl ionic organohydrogels were prepared. The introduction of glycerol and $\mathrm{NaCl}$ rendered the organohydrogel good toughness, high conductivity, adhesiveness, stability in a wide temperature range and self-healing ability. Benefiting from these advantages of the ionic organohydrogel, a flexible all-solid-state supercapacitor and a strain sensor were fabricated separately and their integrated system was obtained finally. The sensor could be driven by the all-solid-state supercapacitor. The selfpowered system could be attached to human skin and precisely detected human motion. This study opens the door to fabricating self-powered systems of strain sensors and flexible all-solid-state supercapacitors by exploiting high-performance ionic organohydrogels as the key material for artificial human motion strain sensors and all-solid-state supercapacitors.
\end{abstract}

\section{Introduction}

Wearable/flexible intelligent devices have been of profound importance in the Internet of Things (IoT) field. ${ }^{1,2}$ With the rapid development of human-machine interface technologies, wearable strain sensors have been widely employed for human activity monitoring. ${ }^{3,4}$ Wearable sensor devices such as epidermal sensors, implantable sensors and real-time monitoring sensors rely on intimate contact between the sensor and the surface of physiological systems with high stability operating under large scale strain. However, most of the conventional sensors based on semiconductors and metals suffer from low sensitivity and a poor sensing range of strain gauges due to their intrinsic metal properties..$^{5-8}$ In that case, many researchers managed to improve the performance of sensors by employing soft polymers as conducting components to fabricate flexible strain-sensing materials. ${ }^{9,10}$ This suggests that the development of effective 
polymeric conductors to achieve high conductivity, superior stretchability and robustness is essentially required for wearable electronic devices.

Ionic conductive hydrogels, typical swollen viscoelastic flexible polymer networks with a large amount of absorbed water which could provide efficient ion transporting channels, may act as a promising candidate. ${ }^{11,12}$ As a kind of conductive polymer, hydrogel electrolytes are usually multi-functional, ${ }^{13-15}$ thus demonstrating advantages over other conductors in the application of flexible devices. ${ }^{12,16-19}$ Various novel hydrogel strain sensors have been fabricated and exhibited high sensitivity, long durability, and outstanding wearable capability for detection of human motion. ${ }^{20-25}$ Nevertheless, these hydrogel-based sensors are unable to work without an external energy storage device, which may result in bulky devices and serious limitations for practical application. ${ }^{26,27}$ Self-powered sensory systems are a promising alternative strategy to promote the use of sensing devices because they are able to acquire power from integrated energy storage devices and operate without an external power source. $^{28-31}$ Thus, hydrogel-based strain sensors, which operate by converting mechanical deformation to an electronic signal, require integration with energy providing devices, such as batteries and supercapacitors. ${ }^{32-35}$ Recently, flexible all-solidstate supercapacitors have attracted much attention in power provision, due to their merits such as long life-time, reliable safety and high power density. ${ }^{36}$ Moreover, all-solid-state supercapacitors also manifested intrinsic safety such as the ability to undergo large mechanical deformation and damage during the application process. In particular, because of the capability to fulfil dual roles of the electrolyte and separator, a superior hydrogel electrolyte is the key factor for fabricating flexible all-solid-state supercapacitors. ${ }^{37,38}$ Therefore, rational design of multifunctional hydrogel electrolyte materials is expected to be an effective approach to simultaneously acquire a highperformance hydrogel sensor, an all-solid-state supercapacitor and their integrated system.

As we know, there are some problems to be confronted with ionic hydrogel electrolytes: malfunction under low temperature, ${ }^{39}$ the balance of ionic conductivity and toughness, ${ }^{40,41}$ difficulty of firmly adhering to various material surfaces, ${ }^{24,41-43}$ deficient reparability, and rupture caused by losing water at high temperature or room temperature for a long time. ${ }^{4,45}$ In detail, most hydrogel electrolytes would inevitably freeze at low temperature and lose water at high temperature due to a large amount of water in the hydrogel. ${ }^{12,43}$ This would largely limit the application temperature range of the hydrogel or increase the sealing process. On the one hand, the low adhesiveness would increase the interfacial resistance between the hydrogel electrolyte and the electrode. ${ }^{46}$ Additionally, they are also hard to directly attach to human skin or clothes as wearable devices. On the other hand, current self-healing hydrogel electrolytes often display limited strength, unsatisfactory healed conductivity and long healing time. However, it is still a puzzle to obtain a remarkable ionic hydrogel electrolyte coupling these properties simultaneously. Strategies to prepare more suitable ionic hydrogels for flexible devices are extremely needed.
In this study, we prepared a high-performance poly(vinyl alcohol)/poly(acrylic amide-acrylic acid)/glycerol/NaCl (PVA/PAMAA/Gly/ $\mathrm{NaCl}$ ) organohydrogel electrolyte with a hybrid physically and chemically cross-linked network. The organohydrogel electrolyte was then used to fabricate a strain sensor and an all-solid-state supercapacitor, respectively. As the functional and key fraction, the PVA/PAMAA/Gly/NaCl organohydrogel electrolyte had high stability in cold and hot environments, high ionic conductivity, good adhesiveness to various substrates, and toughness simultaneously. The assembled organohydrogel strain sensor demonstrated remarkable sensing performance with high sensitivity $(\mathrm{GF}=8.303)$ and reliability to stretch over a wide strain range. The supercapacitor showed a large specific capacitance $\left(75.75 \mathrm{mF} \mathrm{cm}^{-2}\right)$, superior capacitance retention (99.5\% capacitance after 4500 continuous cycles) and excellent electromechanical stability under deformation. By integrating these flexible devices, the sensor could be driven by the supercapacitor. This flexible system could be directly attached on the human body as a health monitoring device and successfully detect full range human motion with high adhesiveness. To the best of our knowledge, this is the first time that an organohydrogel electrolyte based strain sensor and supercapacitor were assembled as a flexible self-powered system. This research opens up opportunities for the design and fabrication of next-generation wearable and portable electronics based on ionic organohydrogel electrolytes.

\section{Results and discussion}

\section{The design principle of the self-powered integrated system}

The fabrication process of the flexible self-powered integrated system is schematically illustrated in Fig. 1, which consisted of constructing an organohydrogel strain sensor and an all-solid-state supercapacitor. Both the functional fraction and the energy storage component were based on the PVA/PAMAA/Gly/ $\mathrm{NaCl}$ organohydrogel electrolyte. As shown in Fig. 1a, the organohydrogel electrolytes were synthesized with PVA and PAMAA as the polymeric networks by free radical in situ polymerization under UV light via a one-pot method. The hydroxyl group of PVA chains and amide group on PAMAA chain would form hydrogen bonds and give a strong physically cross-linked network by rearrangement of macromolecular chains. $\mathrm{NaCl}$ was introduced into the hydrogel and would cause the salting-out effect to enhance electrostatic interactions, contributing to the toughness of the hydrogel. ${ }^{47}$ Moreover, glycerol in the organohydrogel electrolyte would render the organohydrogel electrolyte other prominent properties such as stability in the environment, adhesiveness and self-healing ability as discussed below. Herein, on the basis of the organohydrogel electrolyte, we assembled a stretchable organohydrogel strain sensor by using ITO/PET as the conductive sensing channel without any encapsulation (Fig. 1b). Fig. 1c presents the assembly of a stacked flexible supercapacitor using the synthesized organohydrogel as the separator and electrolyte and with an as-prepared MWCNT film as the electrode. Since the synthesized MWCNT film was vulnerable due to its high modulus and ultra-thin thickness, ${ }^{48}$ it was encapsulated with the PDMS elastomer for protection, 

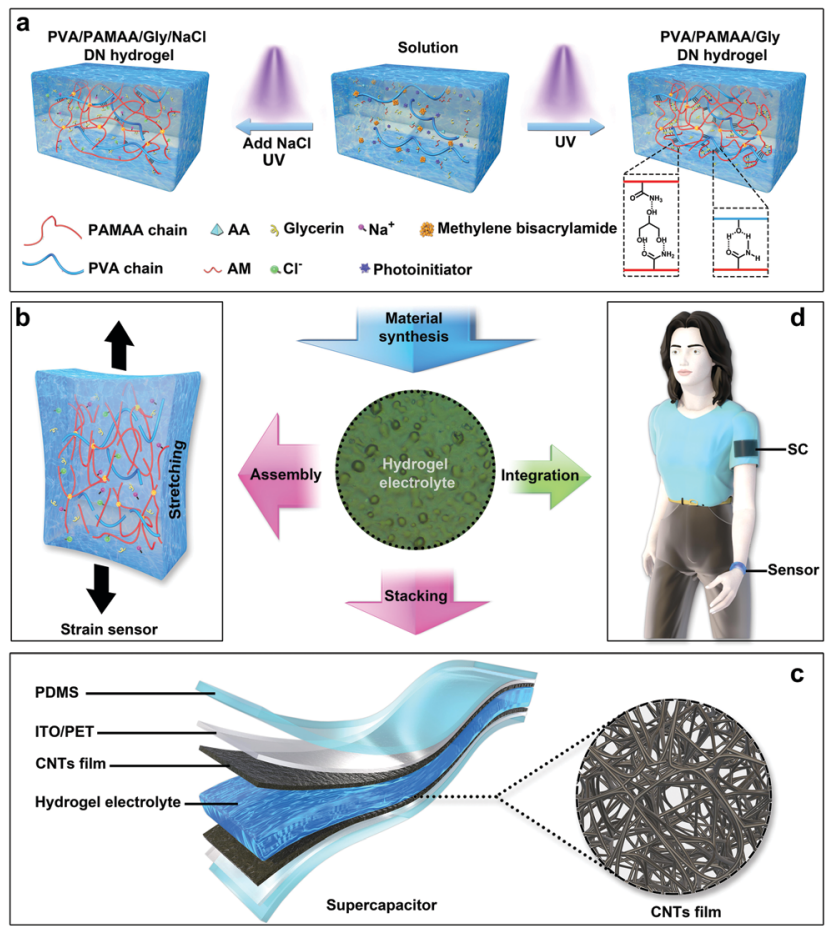

Fig. 1 (a) Schematic illustration of the preparation of the PVA/PAMAA/ $\mathrm{Gly} / \mathrm{NaCl}$ organohydrogel electrolyte. Fabrication of (b) an organohydrogel strain sensor, and (c) a flexible all-solid-state supercapacitor with a CNT film electrode. (d) Integration of the wearable strain sensor and supercapacitor based on the organohydrogel electrolyte attached on a human body.

and a flexible all-solid-state supercapacitor for energy storage was fabricated with an ITO/PET current collector on the top and bottom. Then, once the flexible supercapacitor was fully charged, it could be powerful enough to drive the strain sensor as a wearable integrated system to detect human activities in real-time as shown in Fig. 1d.

\section{Preparation and characterization of the ionic organohydrogel}

In this study, PVA/PAMAA/Gly/NaCl organohydrogel electrolytes with a $\mathrm{NaCl}$ weight of $0,0.14,0.42$ and $0.7 \mathrm{~g}$ were prepared and denoted as PPGN-0, PPGN-1, PPGN-2 and PPGN-3, respectively. The ionic organohydrogel with a higher concentration of $\mathrm{NaCl}$ possessed more uniform and dense pores as shown in Fig. 2a and Fig. S1 in the ESI. $\dagger$ The 3D porous and dense microstructure was attributed to the highly cross-linked physical network. Besides, the formation of a dense porous structure increased the porosity of the organohydrogels, which could absorb more $\mathrm{Na}^{+} / \mathrm{Cl}^{-}$ions and provide more conductive network paths to facilitate ion migration. ${ }^{27,31}$

XRD measurements were carried out to research the crystalline structure change of the organohydrogel electrolyte. As shown in Fig. 2b, the crystalline peak shifted to the right and the peak area decreased when the $\mathrm{NaCl}$ dose rose. This certified that the crystallization of the organohydrogel molecular chain was undermined by $\mathrm{NaCl}$. The crystal peak of $\mathrm{NaCl}$ was absent in the XRD curves of the PVA/PAMAA/Gly/NaCl organohydrogel electrolytes, evidencing that no $\mathrm{NaCl}$ aggregation or crystals existed in the organohydrogels. This was ascribed to the fact that water and glycerol were the binary solvent for the hydrogel electrolytes, and it could prevent the crystallization of $\mathrm{NaCl}$. This also could prove that the PVA/PAMAA/Gly/NaCl organohydrogel electrolyte was homogeneous. The good dispersion of $\mathrm{NaCl}$ as $\mathrm{Na}^{+}$and $\mathrm{Cl}^{-}$ions also would contribute to the high ionic conductivity of the hydrogel electrolyte.

FT-IR spectroscopy was performed to analyze the chemical bonding of the organohydrogel. As shown in Fig. 2c, the band at around $1662 \mathrm{~cm}^{-1}$ was attributed to the amide vibration $\left(\delta_{\mathrm{N}-\mathrm{H}}\right)$ in the organohydrogel, and the hydrogen bonding was validated via the typical band shifting from 3289 to $3335 \mathrm{~cm}^{-1} \cdot 46$ The peak position of this band is related to the hydrogen bonding strength and it indicated that the addition of $\mathrm{NaCl}$ would weaken the hydrogen bonding between PVA and PAMAA chains.

The role of $\mathrm{NaCl}$ in the mechanical properties was examined by tension testing and the results are shown in Fig. $2 d-h$. The pristine PVA/PAMAA/Gly organohydrogel showed a tensile strength and elongation at break of $223 \mathrm{kPa}$ and $1330 \%$, respectively. With the addition of $\mathrm{NaCl}$, the tensile strength of the organohydrogel increased from 252 to $345 \mathrm{kPa}$ as the $\mathrm{NaCl}$ content varied from $0.14 \mathrm{~g}$ to $0.7 \mathrm{~g}$. Meanwhile, the Young's modulus of the organohydrogel increased from 294 to $538 \mathrm{kPa}$ due to the chain entanglement caused by the salting-out effect, resulting in higher stiffness and lower elongation at break (from 1235 to $1002 \%)$. Although the elongation at break decreased with the addition of $\mathrm{NaCl}$, it still was highly stretchable. One also should pay attention to an increase of the toughness from $1.9 \mathrm{MJ} \mathrm{m}^{-3}$ to $2.3 \mathrm{MJ} \mathrm{m}^{-3}$, indicating that the enhancement of the $\mathrm{NaCl}$ content can dissipate more energy to toughen the hydrogels.

A series of strain cycling tests were employed to characterize the self-recovery properties of the PPGN-3 organohydrogel. As presented in Fig. S2a in the ESI, $\dagger$ the consecutive loadingreloading curves were partially overlapped, indicating that the sample underwent a partial self-recovery process. When the strain increased from 100 to $800 \%$, the dissipated energy sharply increased from 18.82 to $399.23 \mathrm{~kJ} \mathrm{~m}^{-3}$, and the calculated dissipation coefficient remained $48.04 \%$ (Fig. S2b in the ESI $\dagger$ ). Fig. S2c and d in the ESI $\dagger$ indicate that the hysteresis loop of the organohydrogel gradually became larger as the resting time increased, and PPGN-3 could recover its energy dissipation (toughness) of $65.28 \%$ after a resting time of $1 \mathrm{~min}$. Clearly, the toughness recovery rates substantially increased with a short resting time and kept a stable value, suggesting that the internal damage of the networks can be rapidly recovered and this can occur within $1 \mathrm{~min}$. As Fig. S2e and $\mathrm{f}$ in the $\mathrm{ESI} \dagger$ reveal, the dissipated energy remained almost unchanged at around $25 \mathrm{~kJ} \mathrm{~m}^{-3}$ after the first loading-unloading cycle, indicating that the uniform and reversible crosslinking structure endued the organohydrogel with exclusive mechanical durability.

In addition, the organohydrogel would obtain anti-freezing ability. It was visually demonstrated that the PPGN-3 organohydrogel electrolyte could be easily stretched to more than 5 times its original length without fracture and maintain 
$\mathbf{a}$

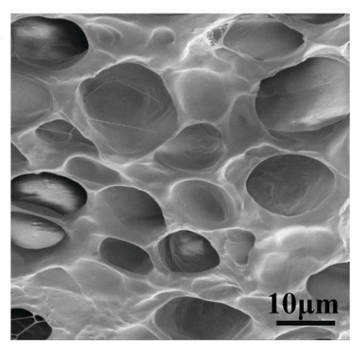

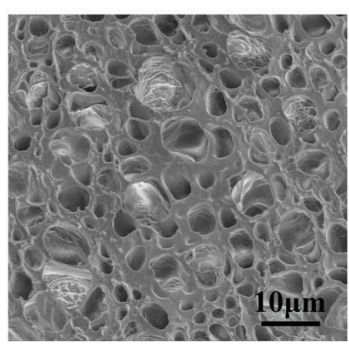

b

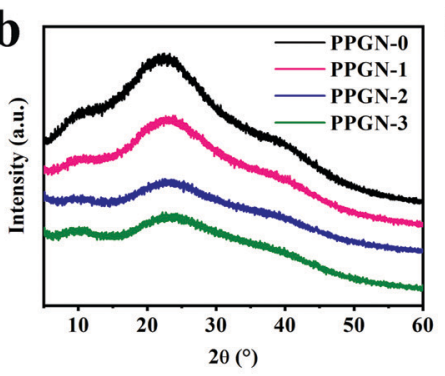

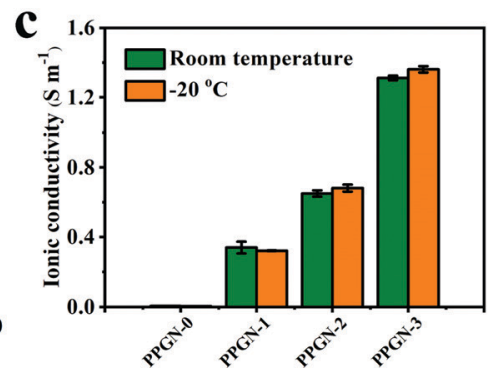

d
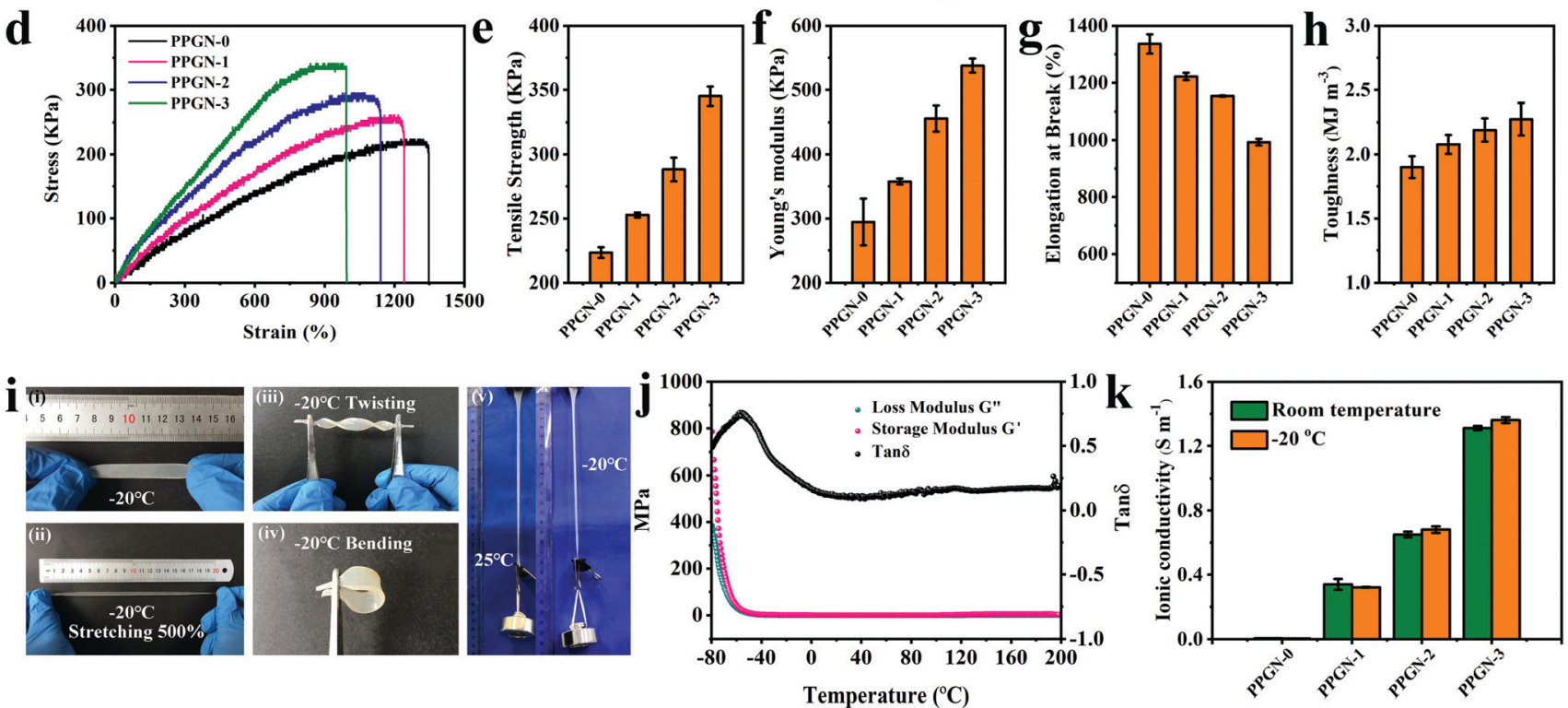

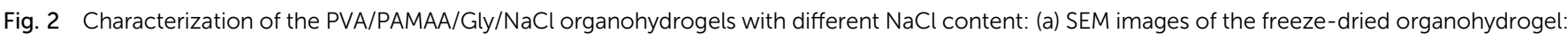

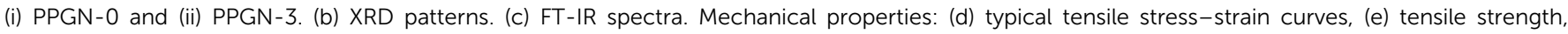

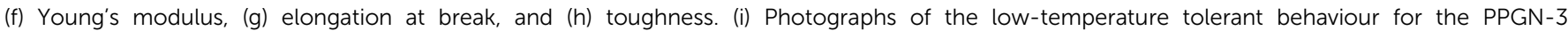

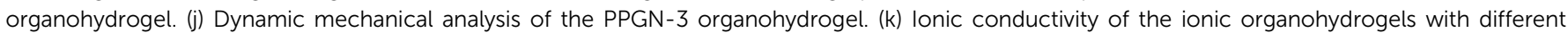
$\mathrm{NaCl}$ content at room temperature and $-20{ }^{\circ} \mathrm{C}$.

excellent reversible stretching, twisting, and bending ability even after storing at $-20{ }^{\circ} \mathrm{C}$ for $24 \mathrm{~h}$ (Fig. 2i). The quantified mechanical properties of the PPGN-3 organohydrogels at $-20{ }^{\circ} \mathrm{C}$, including the tensile strength and elongation at break, were slightly better than those at room temperature (Fig. S3 in the ESI $\dagger$ ). This is mainly attributed to the multiple hydrogen bonds formed between polymer chains and glycerol at $-20{ }^{\circ} \mathrm{C}$, which enhanced the physical cross-linking interaction and enhanced the mechanical properties of the organohydrogel. ${ }^{49,50}$ From the DMA curves (Fig. 2j), the glass transition temperature $\left(T_{\mathrm{g}}\right)$ of the PPGN-3 organohydrogels was $-58.6^{\circ} \mathrm{C}$ and the storage and loss moduli of the organohydrogel electrolyte were smooth and stable without significant change in the temperature range of -60 to $200{ }^{\circ} \mathrm{C}$. The melting point temperature of PPGN-0 was $108.9{ }^{\circ} \mathrm{C}$, while it increased to $126.4{ }^{\circ} \mathrm{C}$ for PPGN-3 (Fig. S4 in the ESI $\dagger$ ). Interestingly, for all organohydrogels, no obvious exothermic peak was observed even when the temperature was decreased to as low as $-80{ }^{\circ} \mathrm{C}$. It demonstrated the outstanding anti-freezing capability of the organohydrogels. The high melting and low freezing point were mainly attributed to the existence of glycerol and its synergistic effect with $\mathrm{NaCl}$. These results also proved that the excellent anti-freezing property of the DN hydrogel had endowed the DN organohydrogel with flexibility in hypothermia. Additionally, as shown in Fig. $2 \mathrm{k}$ and Fig. S5a and b in the ESI, $\dagger$ the ionic conductivity increased with the increase of the $\mathrm{NaCl}$ content at both room temperature and $-20{ }^{\circ} \mathrm{C}$. This was ascribed to the increased concentration of $\mathrm{Na}^{+}$and $\mathrm{Cl}^{-}$ions in the organohydogel matrix. Meanwhile, the conductivity of the PPGN-3 organohydrogel remained $13.14 \mathrm{mS} \mathrm{cm}{ }^{-1}$ after storing at $-20{ }^{\circ} \mathrm{C}$ for $24 \mathrm{~h}$, which was close to the value at room temperature. The high ionic conductivity and good thermomechanical stability expanded the potential application of the organohydrogel.

Similar to adhesive tape, the resultant hydrogel had long-term and repeatable adhesiveness to various substrates. The PPGN-3 organohydrogel could tightly adhere to both hydrophilic and hydrophobic substrate surfaces, such as steel, stone, copper, ceramic, glass, rubber, and wood. Besides, the organohydrogel showed superb tissue-adhesive behaviour on the skin of a volunteer's finger without any residue when peeled off (Fig. 3a). As illustrated in Fig. S6a in the ESI, $\dagger$ the adhesion force of the PPGN-3 organohydrogel was tested using the lap shear test method. ${ }^{51}$ Representative curves and the adhesion strength of the organohydrogel on related surfaces are quantified in 


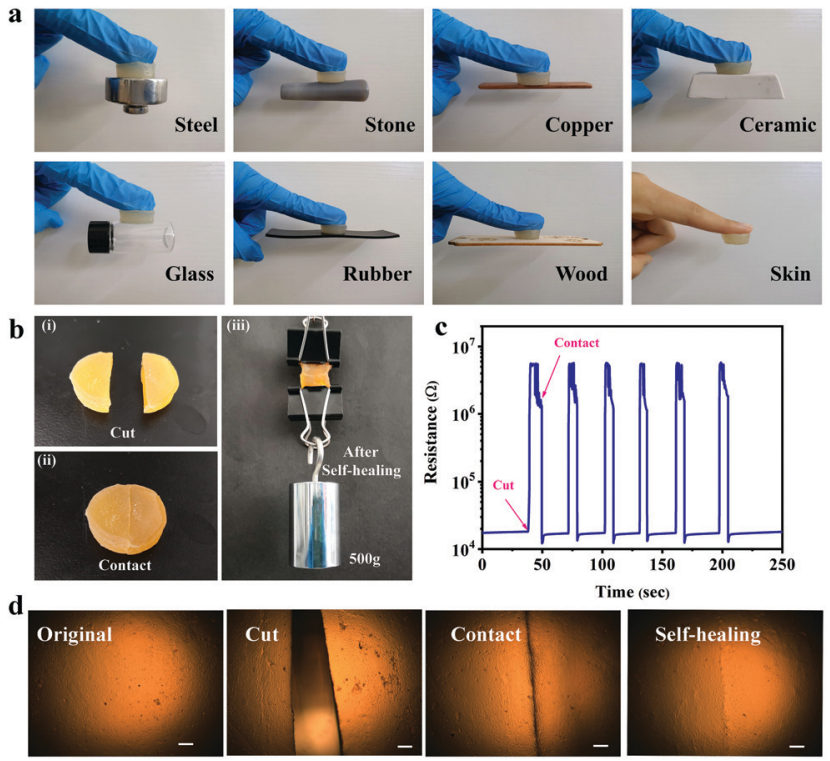

Fig. 3 (a) Display of the adhesive property of the PPGN-3 organohydrogel to various kinds of matrix. (b) The self-healing behaviors between two halves of the PVA/PAMAA/Gly/NaCl organohydrogel: (i) completely bifurcated, (ii) contact, and (iii) stretched after self-healing. (c) Time evolution of the healing process for the PVA/PAMAA/Gly/ $/ \mathrm{NaCl}$ organohydrogel by real-time resistance measurement. (d) Optical microscopic images of the healing process for a PVA/PAMAA/Gly/NaCl organohydrogel being cut and treated by heating. The scale bar is $500 \mu \mathrm{m}$.

Fig. S6b and $\mathrm{c}$ in the ESI, $\uparrow$ to further quantitatively investigate the adhesive performance between the organohydrogel and different substrates. The highest adhesion strengths were $25.30 \mathrm{kPa}$ for aluminium, $11.07 \mathrm{kPa}$ for rubber, $9.18 \mathrm{kPa}$ for copper, and $11.16 \mathrm{kPa}$ for wood. As displayed in Fig. S6d in the ESI, $\dagger$ the adhesion strength remained consistent even after 10 repeated stretching/adhering cycles for aluminum, exhibiting good adhesive performance for repeatable usage. The prominent adhesiveness of the PVA/PAMAA/Gly/NaCl organohydrogels was produced by the unique glycerol-water binary system. Glycerol enhanced the intermolecular interactions in the cross-linked polymer network, thus improving the cohesion of the organohydrogel. As schematically shown in Fig. S6e and $\mathrm{f}$ in the ESI, $\dagger$ the $-\mathrm{OH}$ and $-\mathrm{NH}_{2}$ groups from PAMAA/PVA in the organohydrogel could interact with the $-\mathrm{C}=\mathrm{O},-\mathrm{COOH}$, and $-\mathrm{NH}_{2}$ groups of various material surfaces through hydrogen bonding. Furthermore, the organohydrogel could generate metal complexation with metal ions on the solid surface because of the existence of the $-\mathrm{OH}$ and $-\mathrm{NH}_{2}$ groups from PAMAA and PVA chains, and formed strong interfacial interactions. $^{52,53}$

The self-healing behaviour of the organohydrogel was observed by direct visual inspection. As presented in Fig. 3b, a column-shaped specimen was cut into two halves. Then, they were brought into contact and placed into an oven at $40{ }^{\circ} \mathrm{C}$ for $4 \mathrm{~h}$. During the heating process, the rearrangement of the polymer matrix occurred and the broken interface repaired. Therefore, the separated pieces were repaired, and the healed organohydrogel had sufficient strength to bear a load of $500 \mathrm{~g}$ weight without breaking at the interface. As a comparison in
Fig. S7 in the ESI, $\dagger$ both the tensile strength and elongation at break of the organohydrogels decreased after the self-healing process. However, the healed organohydrogels still maintained a high tensile strength and elongation at break of $283 \mathrm{kPa}$ and $363.24 \%$, respectively. It demonstrated that the organohydrogel electrolytes possessed efficient self-healing ability as well as good mechanical properties after the healing process. Fig. 3c presents six successive breaking-healing processes at the same location. When the sample was cut with a knife, the open circuit resulted in the resistance going to infinity. Then, as the two pieces were contacted, the resistance dropped quickly and remained relatively stable during the cutting-healing cycle. As presented in Fig. S8 in the ESI, $\uparrow$ the resistance reached an initial value within $2.6 \mathrm{~s}$, exhibiting a rapid and repeatable restoring property. Moreover, Fig. 3d illustrates the microscope images of the self-healing process of the organohydrogel. The resultant scar could be healed immediately at $40{ }^{\circ} \mathrm{C}$.

In general, glycerol could permit non-volatility and remarkable thermal tolerance for long-term storage stability. The water-retaining ability of the organohydrogel was evaluated by weight ratio measurements (calculated from $W_{\mathrm{t}} / W_{0}$, where $W_{\mathrm{t}}$ is the instantaneous sample weight, and $W_{0}$ is the original sample weight). The weight of the organohydrogel was recorded at varying time intervals. In a normal environment (Fig. S9a in the ESI $\dagger$ ), the weight of the resultant columnar organohydrogel had a small change with a humidity change. The weight ratio retention of PPGN-3 was $99.7 \%$ after 30 days; in contrast, the hydrogel without glycerol could only keep $52.7 \%$ of its original weight. As illustrated in Fig. S9b in the ESI, $\uparrow$ the organohydrogel with glycerol could impede hydrogel xerosis under high temperature. The residual weight ratio after 30 days $\left(40{ }^{\circ} \mathrm{C}\right)$ was $81.8 \%$, while it dropped to $44.8 \%$ for the hydrogel without glycerol and $\mathrm{NaCl}$. Besides, with the addition of $\mathrm{NaCl}$, the water retention of the organohydrogels increased gradually during the whole storage period, and the resultant organohydrogel remained moist throughout the storage period. The long-term stability of the organohydrogels was due to the presence of glycerol in the polymer network, which was nonvolatile in a normal environment. ${ }^{54}$ Furthermore, the binary system of water and glycerol could prevent the evaporation of water. Therefore, the PVA/PAMAA/ $\mathrm{Gly} / \mathrm{NaCl}$ organohydrogels could maintain the initial state and properties for a long time.

\section{Electromechanical response of the organohydrogel strain sensor}

From the above discussion, it could be found that the PPGN-3 organohydrogel electrolyte exhibited moderate properties of ionic conductivity (Fig. S5c and d in the ESI $\dagger$ ), toughness and long-term durability. Thus, PPGN-3 was used to fabricate a selfpowered integrated system of a strain sensor and an all-solid-state supercapacitor. Movie S1 in the ESI $\dagger$ shows the organohydrogel connected to a closed-circuit equipped with a light-emitting diode (LED). The LED dimmed significantly when the organohydrogel was stretched from $0 \%$ to $400 \%$, indicating a change of the conductivity with stretching. Therefore, this ionic conductive organohydrogel possessed piezoresistive strain dependency. 
As a novel stretchable conductive organohydrogel, this capability of converting mechanical deformation into a detectable electrical signal could be utilized for strain sensing.

As presented in Fig. 4a, the relative resistance change $\left(\Delta R / R_{0}\right)$ increased linearly $\left(R^{2}=0.99\right)$. Firstly, the GF was 1.817 in the strain range of $0-200 \%$, and it increased slightly to 3.436 for the strain range of $200-500 \%$. Furthermore, the sensor displayed an exponential dependency upon the applied strain with a high GF up to 8.303 in the strain range of $500-1000 \%$, which was substantially higher than that of other reported hydrogel based strain sensors (Table S2 in the ESI $\dagger$ ). ${ }^{19-21,23,25,45,47,55-60}$ As demonstrated above, applying strain to the organohydrogel sensor caused a significant change in the electrical resistance signals. In detail, $\mathrm{Na}^{+}$and $\mathrm{Cl}^{-}$ions that absorbed on the polymer chain network provided more dissociable ions. Owing to the existence of strong electrolyte $\mathrm{NaCl}$, a large number of ions were released, which endowed the organohydrogel with strain sensitivity. When the organohydrogel sensor underwent an external force, the porous 3D network structure was damaged, and the transmission path of ions decreased. ${ }^{14}$ Additionally, as illustrated in Fig. S10 in the ESI, $\dagger$ the organohydrogel showed a clearly increased length and decreased cross-section area upon stretching, resulting in an increase in the electric resistance of the
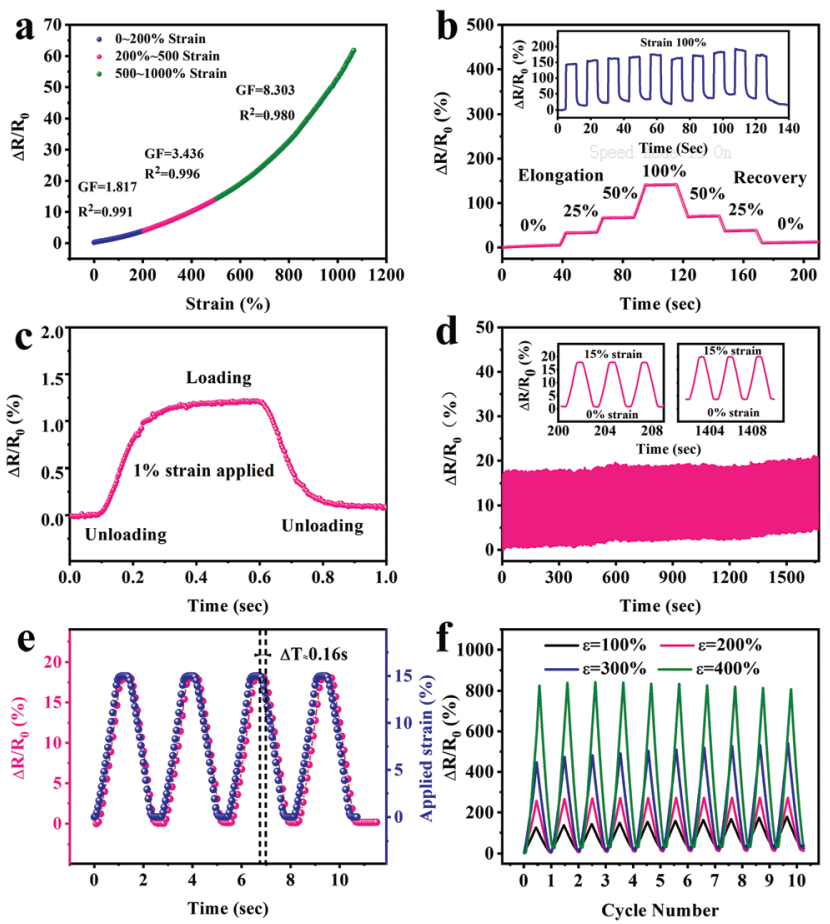

Fig. 4 (a) Relative resistance-strain response curves of the PPGN-3 organohydrogel strain sensor, (b) resistance response of the organohydrogel strain sensor stretched to $25 \%, 50 \%$, and $100 \%$, (c) resistance response of the organohydrogel strain sensor upon loading and unloading a strain of $1 \%$, (d) resistance response of the organohydrogel strain sensor with applied tensile strain between 0 and $15 \%$ for 600 cycles, (e) time-resolved responses of the relative resistance change of the organohydrogel strain sensor upon various strains, and (f) resistance response of repeated stretching tests of the organohydrogel strain sensor at different strains (100\%, 200\%, 300\% and 400\%). organohydrogel strain sensor. Both these phenomena proved the excellent strain sensitivity of the organohydrogel sensor. ${ }^{56}$

Then, a stair-type strain was applied to the sensor, and the results are shown in Fig. $4 \mathrm{~b}$. It was obviously shown that $\Delta R / R_{0}$ increased with a step-like trend during a step by step stretchingholding process. Notably, the resistance of the organohydrogel exhibited a stable plateau state during the holding process. Besides, $\Delta R / R_{0}$ remained almost unchanged at the same tensile strain, indicating excellent electromechanical stability. Particularly, our hydrogel sensor could experimentally detect a low limit of deformation, for example, $1 \%$ tensile strain. To demonstrate the outstanding sensitivity, the sensor was shown to be capable of reliably detecting ultra-small strain as shown in Fig. 4c. Originally, $\Delta R / R_{0}$ approached 0 at a strain of $0 \%$, and then a fast and distinguishable stability response was successfully acquired for both the stretching and releasing process.

During the long-term durability test process, the resistance variation was tracked in ambient conditions without extra sealing. The organohydrogel sensor exhibited high durability and repeatability as shown in Fig. $4 d$. It is worth noting that the sensor generated instantaneous output signal responses during cyclic stretching with great stability and prominent reproducibility after 600 cycles at $15 \%$ strain. Compared with the initial and final 3 cycles (Fig. $4 \mathrm{~d}$ inset), the relative resistance change of the strain sensor had no noticeable deterioration, and only small hysteresis variations were observed. In contrast to most reported hydrogelbased sensors suffering from drying that would cause uneven device performance, ${ }^{44,45,61}$ the as-prepared sensor displayed remarkable non-volatility for long-term durability.

To examine the response time of our sensors to external deformation, the output electrical signals were compared with the dynamic cyclic strain input at a frequency of $0.5 \mathrm{~Hz}$ (Fig. 4e). There was an almost perfectly linear relationship between $\Delta R / R_{0}$ and the applied strain during the tests. The $\Delta R / R_{0}$ waves were almost synchronous with the applied strain under $15 \%$, while a $0.16 \mathrm{~s}$ delay was observed, evidencing that this organohydrogel strain sensor could respond almost immediately to external strain. The porous structure of the organohydrogel sensor was highly beneficial to the sensitivity and in favor of a rapid response. This slight hysteresis may originate from the viscoelastic effects during the stretching-releasing process. ${ }^{62,63}$

Considering extreme deformation conditions, different maximum applied strains of 100, 200, 300 and 400\% were applied to the organohydrogel sensor to assess the repeatability and reliability of the sensing capability under a wide strain range. As illustrated in Fig. 4f, a series of cyclic stretching with a constant strain was carried out to obtain the relative resistance change with fluctuating strain. The time $-\Delta R / R_{0}$ response curves presented high linearity and repeatability. Next, we performed multiple cycle tests by repeated stretching and relaxing of the strain between $100 \%$ and $300 \%$ for 100 cycles (Fig. S11 in the $\mathrm{ESI} \dagger$ ). After dozens of cycles, the electrical signals transferred to a higher relative resistance. This may be due to the cracking of the network structure under stretching, which would be more prominent under a large deformation. However, these observations suggested that the measurement was highly stable and repeatable 
with a large strain cyclic mechanical load. Fig. S12 in the ESI $\dagger$ gives the electromechanical performance of the organohydrogel sensor in response to different tensile rates. As the electronic signals were dependent on the applied tensile rate (from 50 to $500 \mathrm{~mm} \mathrm{~min}^{-1}$ ), the organohydrogel-based strain sensor ensured a reliable response for practical applications.

Furthermore, after storing at $-20{ }^{\circ} \mathrm{C}$ for $24 \mathrm{~h}$, the strainsensitivity of the hydrogel-based strain sensor leveled off and thus it could be applied for sensing at subzero temperatures (Fig. S13 in the ESI $\dagger$ ). Table S2 in the ESI $\dagger$ shows the comparison of the properties of our device with other reported studies. ${ }^{19-21,23,25,45,47,55-60}$ By considering the overall performance and unique characteristics, this PVA/PAMAA/Gly/NaCl organohydrogel sensor is a promising material for realizing a wearable integrated sensory system.

\section{Electrochemical performance of the flexible all-solid-state supercapacitor}

For human-machine interface technology, it is impractical to operate sensing devices via long wires connected to external power. Encouraged by the superior adhesiveness, high ionic conductivity and toughness, we assembled a flexible all-solidstate supercapacitor with the PVA/PAMAA/Gly/NaCl organohydrogel electrolyte. As for the electrode material (Fig. S14 in the ESI $\dagger$ ), an easily prepared MWCNT film with high electronic conductivity $\left(44.7 \mathrm{~S} \mathrm{~cm}^{-1}\right)$ and mechanical strength (20 MPa) was a suitable candidate. As a highly flexible thin film, the MWCNT film could tolerate omni-directional bending without any damage or fracture. Therefore, by combining the selfadhesive organohydrogel electrolyte and flexible MWCNT film electrode with a simple device-assembly strategy based on a hydrophilic interaction and hydrogen bonding mechanism, an all-solid-state supercapacitor was constructed (Fig. S15 in the ESI $\dagger$ ). The electrochemical characteristics of the flexible supercapacitor were assessed by CV, GCD and cycling stability measurements in a two-electrode system. As expected and as shown in Fig. 5a, the CV curves showed a quasi-rectangular shape and mirror-image symmetry at scanning rates ranging from 5 to $100 \mathrm{mV} \mathrm{s}^{-1}$, revealing a prominent rate capability, which can be mainly ascribed to electronic double-layer capacitance. ${ }^{64}$ In addition, the linear profile and symmetric triangular shape of the GCD curves at different current densities ranging from 0.5 to $2.0 \mathrm{~mA} \mathrm{~cm}{ }^{-2}$ and in a potential window of 0 to $1.0 \mathrm{~V}$ are shown in Fig. 5b. This suggested that a typical reversible charging and discharging process occurred at the interface between the electrolyte and electrodes. As a supplement, CV and CGD curves in a $0-1.5 \mathrm{~V}$ potential window are also provided in Fig. S16 in the ESI. $\uparrow$ Such a high operating potential window is superior to the reported flexible supercapacitors with hydrogel based electrolytes. ${ }^{18,65,66}$

The areal capacitance (CA) and coulombic efficiency of the supercapacitor at diverse current densities were calculated and are plotted in Fig. 5c. Although the specific capacitance was delivering a gradual decrease at increasing current densities, the achieved value of $75.75 \mathrm{mF} \mathrm{cm}^{-2}$ at $0.5 \mathrm{~mA} \mathrm{~cm}{ }^{-2}$ was much higher than other reported hydrogel electrolyte based all-solid-state
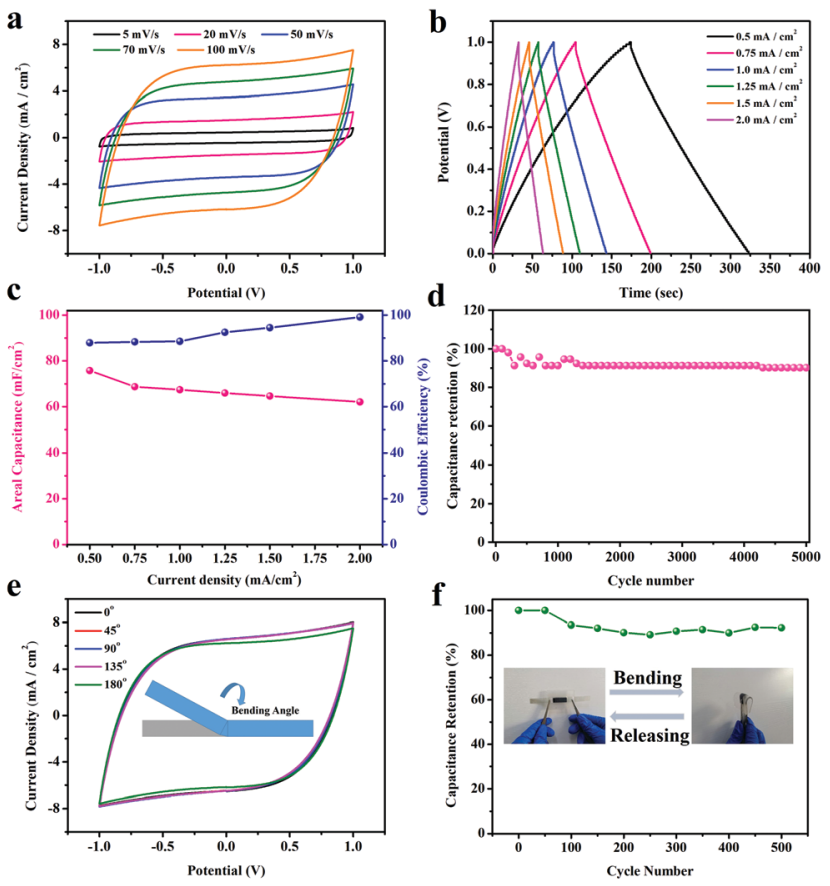

d
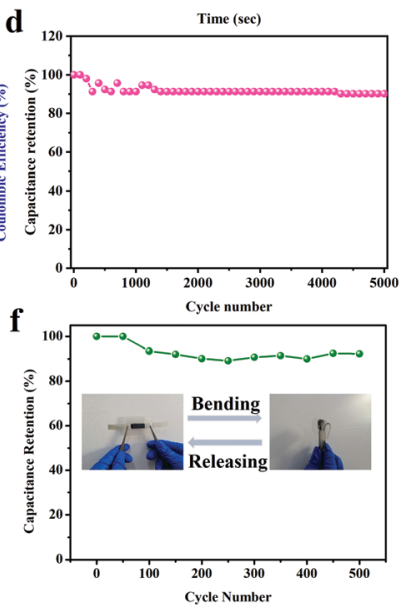

Fig. 5 Electrochemical performance of the supercapacitor. (a) CV curves at scan rates of 5-100 $\mathrm{mV} \mathrm{s}^{-1}$. (b) GCD curves at a current density of $0.5-2 \mathrm{~mA} \mathrm{~cm}^{-2}$. (c) Areal capacitance and Coulomb efficiency at different current densities calculated from the GCD curves. (d) Capacitance retention at $5 \mathrm{~mA} \mathrm{~cm} \mathrm{~cm}^{-2}$ after 5000 charging and discharging cycles. (e) CV curves under different bending states. (f) Specific capacitance retention rate after 500 bending cycles.

supercapacitors (Table S3 in the ESI $\dagger$ ). ${ }^{18,31,38,39,46,64,67-70}$ From Fig. 5c, it can also be observed that the coulombic efficiency increased slightly with the increase of the current density and it was estimated to be $99.1 \%$. In addition, this device had an energy density of $10.52 \mu \mathrm{W} \mathrm{h} \mathrm{cm}{ }^{-2}$ and a power density of $0.25 \mathrm{~mW} \mathrm{~cm}^{-2}$ (Fig. S17 in the ESI $\dagger$ ). The exceptional electrochemical performance was due to the relatively high ionic transport efficiency, the large surface area of the MWCNT film, and the increase of the contact area between the electrolyte and the electrode, which would decrease the interfacial contact resistance and improve the reaction kinetics for prolonged cycling under high current densities. ${ }^{71,72}$ The capacitance retention was $90.2 \%$ after 5000 repeated charge/discharge cycles at a current density of $5 \mathrm{~mA} \mathrm{~cm}{ }^{-2}$ (Fig. 5d). Although a slightly reduced capacitance retention was observed, the supercapacitor displayed superior cycling stability. The main factor can be ascribed to the stable crosslinked network structure and the resulting high mechanical strength providing protection for ion transportation.

Additionally, when integrated into the sensing component for wearable application, supercapacitors are required to be highly flexible, and even to be easily rolled up. The electrochemical stability of the supercapacitor under deformation with various bending angles is displayed in Fig. 5e. The CV curves of the flexible all-solid-state supercapacitor at different bending angles presented negligible distinction in terms of the quasi-rectangular shape at $100 \mathrm{mV} \mathrm{s}^{-1}$. They were quasi-rectangular CV curves and there was no obvious difference in the $\mathrm{CV}$ response under different bending 
radii compared with the initial status. Cyclic bending tests under an angle of $180^{\circ}$ were carried out to investigate the ultimate energy storage performance and stability under flexural strain. As presented in Fig. 5f, the supercapacitor kept up to $92.3 \%$ capacitance retention after bending for 500 repeats, proving its prominent electrochemical reliability, mechanical robustness and potential for power supply devices. This prominent performance was ascribed to the strong bonds between the electrode and the electrolyte preventing delamination or displacement among the multiple layers under mechanical deformation. Moreover, the steady organocrosslinked network endowed high mechanical strength to the solid organohydrogel electrolyte, which prevented fatigue or fracture during long-term operation.

Moreover, the electrochemical performance of the supercapacitors in a low temperature environment appeared to be nearly the same as that at room temperature (Fig. S18 in the ESI $\dagger$ ). Therefore, the fabricated supercapacitors based on the organohydrogel electrolyte possessed extraordinary electrochemical performance, and had potential for developing flexible power storage devices operating in extreme low temperature conditions.

\section{Self-powered integrated system}

Although many hydrogel-based strain sensors and supercapacitors have been developed, their application in portable integrated electronic devices is still challenging. In order to realize the integration target of our devices, the fabricated strain sensor and supercapacitors and an ammeter were connected in series to form a complete circuit. After charging the supercapacitor using an electrochemical workstation with a turn-on voltage, the connected flexible integrated sensor was driven by the charged supercapacitor.

The discharging curve of the supercapacitor (red) and the current versus time plots of the strain sensor driven by the supercapacitor (blue) are provided in Fig. 6a. The electronic signal of both the current and the potential exhibited a similar decreasing tendency because of the energy consumption caused by the self-discharging process. When mechanical strain was applied, the organohydrogel-based strain sensor was stretched, resulting in the dramatic decrease of the current (opposite to resistance). Furthermore, during the holding state, the current of the sensor remained stable. The self-powered strain sensor
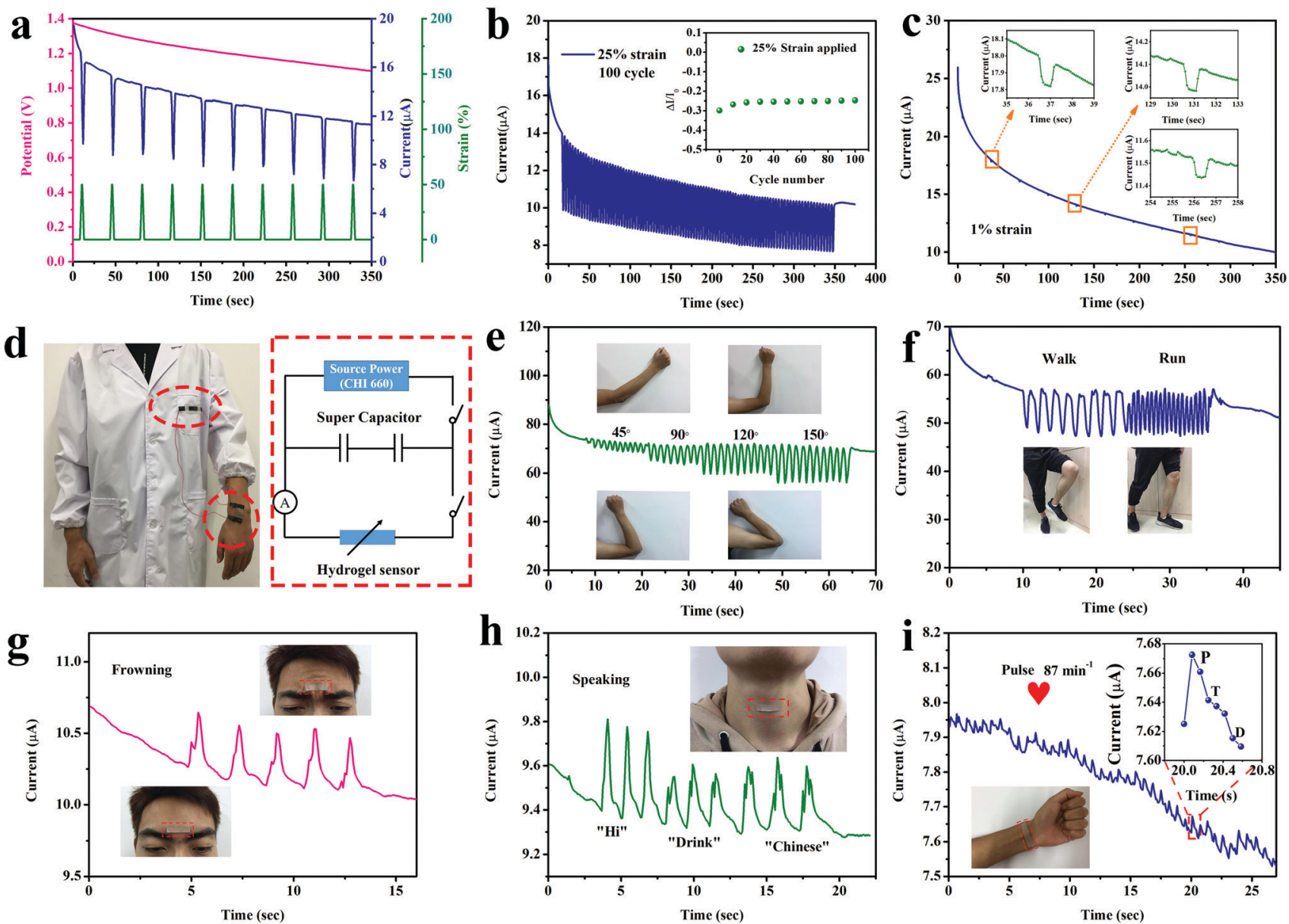

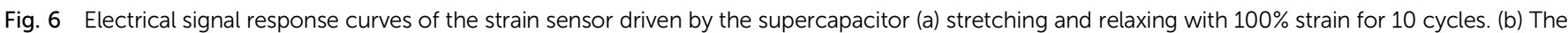

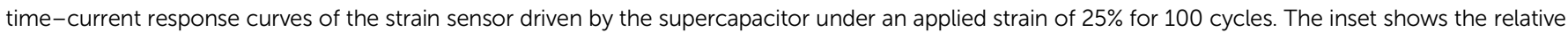

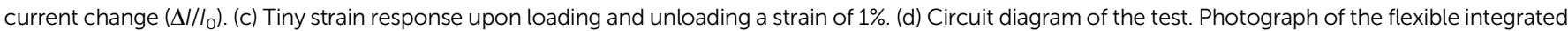

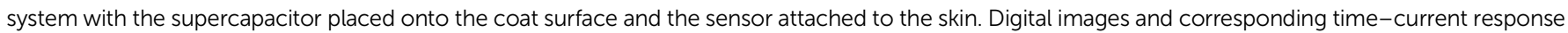
curves of the integrated system in human motion monitoring: (e) arm movement, (f) walking \& running, (g) frowning, (h) speaking, and (i) wrist pulse. 
exhibited excellent sensitivity and stability, which was similar to the result of the sensor operated using an external source. As demonstrated in Fig. S19a in the ESI, $\dagger$ although the current and voltage were dropping continuously with similar trends, the resistance (voltage/current) change of the organohydrogel with the applied loading was constant. The self-discharge behavior with the fabricated supercapacitors is shown in Fig. S19b and c in the ESI. $\dagger$ After 800 seconds, the devices retained a $1.27 \mathrm{~V}$ potential for storage of energy. When the charged supercapacitor drives the strain sensor, the sensor could operate stably for more than 800 seconds. After that, the relative signal drift at $100 \%$ strain of the organohydrogel sensor was acceptable and a clear current response signal was observed, allowing for reliable practical utilization as a self-powered sensory system. ${ }^{63,73}$

To compare different driving voltages, the change of the corresponding current under a 50\% strain is plotted in Fig. S20 in the ESI. $\dagger$ It was noted that the flexible all-solid-state supercapacitor could be charged to a working potential of $0.66 \mathrm{~V}$ and performed stably to operate the sensor. In view of the different conductive mechanisms of conventional piezoresistive sensors, the hydrogel sensor, based on ion conduction, could be operated for a considerably long time ${ }^{30-32,74}$ (Table S4 in the ESI $\dagger$ ). As can be seen from Fig. 6b, upon cyclic stretching and releasing at a constant strain of $25 \%$, the current signal of the sensor changed cyclically and stably. It was obvious that the current decreased. However, the relative current change $\left(\Delta I / I_{0} ; \Delta I\right.$ is the relative current change with strain, $I_{0}$ is the initial current prior to straining) remained almost constant during the 100 cycle loading.

This indicated that using a supercapacitor as a substitute for a conventional energy unit as the driving source was feasible and durable. The ability of the wireless powered wearable organohydrogel strain sensor in detecting various mechanical strains was evaluated. The current-strain response curves, acquired from the integrated supercapacitors, showed that tiny changes of the current signals under $1 \%$ strain were obviously distinguished, and these were magnified as shown in Fig. 6c. Also, as described in detail in Fig. S21 in the ESI, $\dagger$ the strainresponse (current-time) curves confirmed that this flexible integrated system assembly of the supercapacitor and sensor performed stably to drive and operate the electronic device even under a wide strain range (from 5\% to 100\%). The result covered a more wide strain range than most reported selfpowered flexible integrated systems..$^{30-32,34,35,70,75}$ Leveraging its excellent adhesiveness, the organohydrogel epidermal strain sensor could be perfectly attached to human skin directly and withstand the stretching or follow the movement of the body (Fig. S22 in the ESI $\dagger$ ). Furthermore, this integrated self-powered strain sensor was qualified to be used reliably in full range movement monitoring. Therefore, as illustrated in Fig. 6d, the flexible integrated system was attached to the body directly to detect human motion. Fig. 6e illustrates the detection of arm bending with an increase in angle. The attached strain sensor was stretched when the bending angle increased from $45^{\circ}$ to $150^{\circ}$, resulting in an increased maximum value in the current change. During cyclic bending with a certain angle, the self-powered sensor exhibited a stable response. Moreover, the sensors were mounted on the knee joint to monitor low frequency (walking) and high frequency (running) motion processes. As presented in Fig. 6f, the organohydrogel sensor could detect the variation of the current response with different frequencies and could detect the difference of the motion speed. These results demonstrated the satisfactory capability of an integrated system to distinguish the same motion at different frequencies.

In addition to the successful monitoring of the above mentioned large scale motions, the capability of the integrated sensory system to detect subtle motions was further confirmed. When adhered to the middle of the forehead, facial expressions, such as frowning, could be distinguished (Fig. 6g). Subsequently, the integrated sensor system was attached onto the throat of a volunteer to detect the complicated epidermis movements during speaking (Fig. 6h). The current signals changed obviously when the volunteer pronounced the words "hi", "drink" and "Chinese" in sequence. Interestingly, the pronunciation of "Chinese" caused three characteristic peaks, while "hi" and "drink" presented a single and a double peak, respectively. More importantly, remarkable reproducibility, and characteristic peaks and valleys for each signal could be obtained, evidencing great reliability for phonation signal acquisition.

As proof-of-concept for real-time health monitoring, we applied the integrated sensing system to detect the pulse of an adult male in the wrist, which is generally utilized as a personalized healthcare parameter (Fig. 6i). The radial artery frequency (84 pulses $\mathrm{min}^{-1}$ ) could be counted via the current signal variation, demonstrating the subject in good health condition as an adult male. ${ }^{76}$ More importantly, due to the ultra-low detection limit of the integrated sensor, three featured peaks corresponding to percussion (P), tidal (T), and diastolic (D) for the pulse waveforms of the volunteer were accurately resolved. It is worth noting that the current responses of the self-powered sensor system on the forehead, throat and wrist showed an observed opposite tendency due to the opposite movements of muscles caused by stress releasing, which was different from the arm movement and walk and run. ${ }^{77}$ In a word, as we highly expected, the organohydrogel-based strain sensor was successfully driven by the high-performance flexible supercapacitor and had the ability of monitoring the full range of human motions.

\section{Conclusions}

In summary, we have proposed an easy fabrication method for an anti-freezing, adhesive and self-healable ionic organohydrogel electrolyte combining outstanding antifreezing ability, mechanical properties and good ionic conductivity. A strain sensor was fabricated with this hydrogel electrolyte to detect mechanical stress. It exhibited great sensitivity and a wide strain range, a low detection limit of $1 \%$ strain, high durability of 600 cycles of stress loading-unloading, and a fast response time of $0.16 \mathrm{~s}$. Furthermore, the organohydrogel electrolyte was used as a solid electrolyte to develop a flexible all-solid-state supercapacitor with a MWCNT film as the electrode. The assembled flexible supercapacitor showed an areal capacitance of $75.75 \mathrm{mF} \mathrm{cm} \mathrm{cm}^{-2}$ at $0.5 \mathrm{~mA} \mathrm{~cm} \mathrm{~m}^{-2}$, and $92.3 \%$ retention of the capacitance after 
500 bending cycles. Finally, we integrated the flexible all-solidstate supercapacitor with a strain sensor into a self-powered system that could be attached to human skin and precisely detected human motion. These above applications demonstrated that the rational strategy of designing and manufacturing a hydrogel for self-powered strain sensors could provide a promising design to realize multifunctional wearable devices.

\section{Experimental section}

PVA/PAMAA/Gly/NaCl ionic organohydrogels were synthesized by a one-pot method. Firstly, $0.2 \mathrm{~g}$ PVA was dissolved in $4.0 \mathrm{~mL}$ distilled water with stirring at $90{ }^{\circ} \mathrm{C}$. Then $3.0 \mathrm{~g}$ glycerol and a certain proportion of $\mathrm{NaCl}$ were slowly added to PVA aqueous solution (as shown in Table S1 in the ESI $\dagger$ ), and the PVA/Gly/ $\mathrm{NaCl}$ stock solution was obtained. Then $1.9936 \mathrm{~g}$ AM, $0.107 \mathrm{~g} \mathrm{AA}$, $1.36 \mathrm{mg}$ MBA and $0.0662 \mathrm{~g}$ Irgacure 2959 were added into the PVA/ Gly/NaCl solution and stirred in a water bath until all components were dissolved. A reflux device was used to prevent water evaporation during the dissolution process. The stock solution was then slowly poured into a mold of a $2 \mathrm{~mm}$ height silicone spacer combined with two glass plates. Finally, the resulting solution was placed under UV light $(\lambda=365 \mathrm{~nm}$ wavelength, intensity of $8 \mathrm{~W}$ ) for $1 \mathrm{~h}$ to form the PVA/PAMAA/Gly/NaCl organohydrogel electrolyte. The PVA/PAMAA/Gly hydrogel without $\mathrm{NaCl}$ was prepared by the same method.

\section{Preparation of the hydrogel strain sensor}

An ITO/PET film as an electrode was attached on both sides of the organohydrogel, and conductive silver paste was applied to make perfect bonding and electrical contact between the electrodes and the organohydrogel electrolyte.

\section{Fabrication of the all-solid-state supercapacitor}

The flexible all-solid-state supercapacitor was assembled in a symmetric two-electrode configuration by using a MWCNT film as the electrode, and the organohydrogel as both the electrolyte and separator. Then, tin oxide/polyethylene terephthalate (ITO/PET) was taped to the MWCNT film electrode as the current collector through silver paste. Finally, the flexible all-solidstate supercapacitor was obtained with encapsulation by polydimethylsiloxane (PDMS, Dow Corning Sylgard 184).

\section{Characterization and measurement}

The organohydrogel was firstly soaked in excess deionized water for $12 \mathrm{~h}$ to exchange glycerol. ${ }^{49}$ Then the samples were freezedried using liquid nitrogen before drying in a vacuum oven for $36 \mathrm{~h}$. The morphology and microstructure were observed with scanning electron microscopy (SEM, Verios G4, Thermo Fisher Scientific). Meanwhile the microscope images were obtained through a digital microscope (DVM6, LEICA).

The FTIR spectra of the organohydrogel electrolyte were obtained for wavenumbers ranging from $500-4000 \mathrm{~cm}^{-1}$ with a resolution of $4 \mathrm{~cm}^{-1}$.
The XRD patterns of the organohydrogels were measured on an X'Pert PRO (Panalytical, Netherlands). The scanning speed was $0.209^{\circ} \mathrm{s}^{-1}$ with a step size of $0.0167^{\circ}$ and they were recorded in an angle range of $5-60^{\circ}$ at ambient temperature.

For mechanical measurements, the organohydrogel samples were prepared in a dumbbell-shape with a gauge length of $30 \mathrm{~mm}$, a width of $4 \mathrm{~mm}$, and a thickness of $2 \mathrm{~mm}$ for tensile testing at room temperature and $-20{ }^{\circ} \mathrm{C}$ (the samples were placed in a refrigerator set at $-20{ }^{\circ} \mathrm{C}$ for $24 \mathrm{~h}$ and immediately tested in cold conditions). All measurements of the mechanical properties were carried out with an electrical universal materials testing machine (CMT 6503, MTS/SANS, China). The crosshead speed was fixed at $100 \mathrm{~mm} \mathrm{~min}^{-1}$ for the normal tensile tests and the cyclic tensile tests, and a $1 \mathrm{kN}$ force sensor was employed. The change of the mechanical properties with respect to temperature was further investigated using a dynamic thermomechanical analyzer (DMA1, METTLER TOLEDO) in a temperature range from -80 to $200{ }^{\circ} \mathrm{C}$. Each test was repeated at least 3 times.

The ionic conductivity of the organohydrogels at room temperature and $-20{ }^{\circ} \mathrm{C}$ was tested by electrochemical impedance spectroscopy. The ionic conductivity $\sigma\left(\mathrm{mS} \mathrm{cm}^{-1}\right)$ was obtained from the equation below (eqn 1):

$$
\sigma=\frac{R}{L \cdot S}
$$

where $S$ is the cross-sectional area of the hydrogel $\left(\mathrm{cm}^{2}\right)$; $L$ represents the distance between each two electrodes $(\mathrm{cm})$ and $R$ is the bulk resistance $(\Omega)$.

Both the resistance and the applied mechanical strain of the organohydrogel sensors were measured simultaneously and recorded continuously using a digital SourceMeter (Keithley 2450, Tektronix Co., USA) and digital data acquisition system (cDAQ-9174, NI) through the LabVIEW program. The organohydrogel samples were prepared with a gauge length of $30 \mathrm{~mm}$, a width of $4 \mathrm{~mm}$, and a thickness of $2 \mathrm{~mm}$ for electromechanical testing. To avoid a delay between the detectors, the electrical and mechanical signals were synchronously recorded. The sensitivity of the organohydrogel with an increase in strain was investigated. The sensitivity defined as the gauge factor was calculated by the equation (eqn 2 ): ${ }^{20}$

$$
\mathrm{GF}=\frac{\Delta R / R_{0}}{\varepsilon}
$$

where $\Delta R$ is the resistance change with strain, $R_{0}$ is the initial resistance prior to strain, and $\varepsilon$ is the applied strain.

In addition, the performance of the supercapacitor was evaluated by electrochemical impedance spectroscopy (EIS), cyclic voltammetry (CV), and galvanostatic charge/discharge (GCD) techniques through a CHI660 electrochemical workstation (Chenhua Co., China) with a two-electrode configuration. The area specific capacitance $C_{\mathrm{A}}\left(\mathrm{mF} \mathrm{cm}^{-2}\right)$ of the supercapacitors was calculated from the GCD curves according to the following formula (eqn 3):

$$
C_{\mathrm{A}}=\frac{I \times \Delta t}{A \times \Delta V}
$$

where $I$ is the constant discharge current (mA), $A$ is the electrode area $\left(\mathrm{cm}^{2}\right), \Delta t$ is the discharge time (s) and $\Delta V$ is the voltage drop 
during discharge. The energy densities $\left(E_{\mathrm{A}}, \mu \mathrm{W} \mathrm{h} \mathrm{cm}{ }^{-2}\right)$ and power densities $\left.\left(P_{\mathrm{A}}, \mu \mathrm{W} \mathrm{cm}\right)^{-2}\right)$ were calculated by using the following formula (eqn (4) and (5)):

$$
\begin{gathered}
E_{\mathrm{A}}=\frac{C_{\mathrm{A}} \times \Delta V^{2}}{2 \times 3600} \\
P_{\mathrm{A}}=\frac{E_{\mathrm{A}} \times 3600}{\Delta t}
\end{gathered}
$$

\section{Conflicts of interest}

There are no conflicts to declare.

\section{Acknowledgements}

J. R. H., S. J. P. and J. F. G. contributed equally to this work. This research was supported by the Natural Science Foundation of Fujian Province (No. 2017J05023), National Natural Science Foundation of China (No. 21676057 and 11972005), and the NSF for Distinguished Young Scholars of Fujian Province (Grant No. 2017J07004). The volunteers took part in this proof-of-concept demonstration with full understanding of the process and any risks involved. Signed consent was received from the volunteers for their participation in the sensor test experiments.

\section{Notes and references}

1 Z. Wu, Y. Wang, X. Liu, C. Lv, Y. Li, D. Wei and Z. Liu, Adv. Mater., 2019, 31, 1800716.

2 C. Wang, K. Xia, H. Wang, X. Liang, Z. Yin and Y. Zhang, Adv. Mater., 2019, 31, 1801072.

3 Y. R. Jeong, H. Park, S. W. Jin, S. Y. Hong, S. S. Lee and J. S. Ha, Adv. Funct. Mater., 2015, 25, 4228-4236.

4 X. Li, T. Yang, Y. Yang, J. Zhu, L. Li, F. E. Alam, X. Li, K. Wang, H. Cheng, C. Te Lin, Y. Fang and H. Zhu, Adv. Funct. Mater., 2016, 26, 1322-1329.

5 S. Ryu, P. Lee, J. B. Chou, R. Xu, R. Zhao, A. J. Hart and S. G. Kim, ACS Nano, 2015, 9, 5929-5936.

6 H. Zhang, N. Liu, Y. Shi, W. Liu, Y. Yue, S. Wang, Y. Ma, L. Wen, L. Li, F. Long, Z. Zou and Y. Gao, ACS Appl. Mater. Interfaces, 2016, 8, 22374-22381.

7 M. Amjadi, K. U. Kyung, I. Park and M. Sitti, Adv. Funct. Mater., 2016, 26, 1678-1698.

8 C. Wang, X. Li, E. Gao, M. Jian, K. Xia, Q. Wang, Z. Xu, T. Ren and Y. Zhang, Adv. Mater., 2016, 28, 6640-6648.

9 Q. Liu, J. Chen, Y. Li and G. Shi, ACS Nano, 2016, 10, 7901-7906.

10 C. Zhang, H. Li, A. Huang, Q. Zhang, K. Rui, H. Lin, G. Sun, J. Zhu, H. Peng and W. Huang, Small, 2019, 15, 1805493.

11 Y. Zhou, C. Wan, Y. Yang, H. Yang, S. Wang, Z. Dai, K. Ji, H. Jiang, X. Chen and Y. Long, Adv. Funct. Mater., 2019, 29, 1-8.

12 H. Liao, X. Guo, P. Wan and G. Yu, Adv. Funct. Mater., 2019, 29, 1904507.
13 J. Wu, Z. Wu, Y. Wei, H. Ding, W. Huang, X. Gui, W. X. Shi, Y. Shen, K. Tao and X. Xie, ACS Appl. Mater. Interfaces, 2020, 12, 19069-19079.

14 J. Wu, S. Han, T. Yang, Z. Li, Z. Wu, X. Gui, K. Tao, J. Miao, L. K. Norford, C. Liu and F. Huo, ACS Appl. Mater. Interfaces, 2018, 10, 19097-19105.

15 J. Wu, Z. Wu, H. Xu, Q. Wu, C. Liu, B. R. Yang, X. Gui, X. Xie, K. Tao, Y. Shen, J. Miao and L. K. Norford, Mater. Horiz., 2019, 6, 595-603.

16 C. R. Chen, H. Qin, H. P. Cong and S. H. Yu, Adv. Mater., 2019, 31, 1-10.

17 J. S. Wei, C. Ding, P. Zhang, H. Ding, X. Q. Niu, Y. Y. Ma, C. Li, Y. G. Wang and H. M. Xiong, Adv. Mater., 2019, 31, $1-7$.

18 H. Li, T. Lv, H. Sun, G. Qian, N. Li, Y. Yao and T. Chen, Nat. Commun., 2019, 10, 1-8.

19 Y. M. Kim and H. C. Moon, Adv. Funct. Mater., 2019, 1, 1907290.

20 X. Jing, H. Y. Mi, Y. J. Lin, E. Enriquez, X. F. Peng and L. S. Turng, ACS Appl. Mater. Interfaces, 2018, 10, 20897-20909.

21 G. Cai, J. Wang, K. Qian, J. Chen, S. Li and P. S. Lee, Adv. Sci., 2017, 4, 1600190.

22 S. Xia, S. Song and G. Gao, Chem. Eng. J., 2018, 354, 817-824. 23 Y. Z. Zhang, K. H. Lee, D. H. Anjum, R. Sougrat, Q. Jiang, H. Kim and H. N. Alshareef, Sci. Adv., 2018, 4, 1-8.

24 C. Cui, C. Shao, L. Meng and J. Yang, ACS Appl. Mater. Interfaces, 2019, 11, 39228-39237.

25 C. Shao, M. Wang, L. Meng, H. Chang, B. Wang, F. Xu, J. Yang and P. Wan, Chem. Mater., 2018, 30, 3110-3121.

26 R. Fu, L. Tu, Y. Zhou, L. Fan, F. Zhang, Z. Wang, J. Xing, D. Chen, C. Deng, G. Tan, P. Yu, L. Zhou and C. Ning, Chem. Mater., 2019, 31, 9850-9860.

27 J. Xu and G. Shen, Nano Energy, 2015, 13, 131-139.

28 D. Kim, D. Kim, H. Lee, Y. R. Jeong, S. J. Lee, G. Yang, H. Kim, G. Lee, S. Jeon, G. Zi, J. Kim and J. S. Ha, Adv. Mater., 2016, 28, 748-756.

29 Y. Lin, J. Chen, M. M. Tavakoli, Y. Gao, Y. Zhu, D. Zhang, M. Kam, Z. He and Z. Fan, Adv. Mater., 2019, 31, 1-9.

30 W. Li, X. Xu, C. Liu, M. C. Tekell, J. Ning, J. Guo, J. Zhang and D. Fan, Adv. Funct. Mater., 2017, 27, 1-12.

31 H. Park, J. W. Kim, S. Y. Hong, G. Lee, D. S. Kim, J. Hyun Oh, S. W. Jin, Y. R. Jeong, S. Y. Oh, J. Y. Yun and J. S. Ha, Adv. Funct. Mater., 2018, 28, 1-11.

32 Y. Song, H. Chen, X. Chen, H. Wu, H. Guo, X. Cheng, B. Meng and H. Zhang, Nano Energy, 2018, 53, 189-197.

33 C. Zhang, H. Li, A. Huang, Q. Zhang, K. Rui, H. Lin, G. Sun, J. Zhu, H. Peng and W. Huang, Small, 2019, 15, 1-8.

34 Y. Song, H. Chen, Z. Su, X. Chen, L. Miao, J. Zhang, X. Cheng and H. Zhang, Small, 2017, 13, 1-10.

35 L. Yu, Y. Yi, T. Yao, Y. Song, Y. Chen, Q. Li, Z. Xia, N. Wei, Z. Tian, B. Nie, L. Zhang, Z. Liu and J. Sun, Nano Res., 2019, 12, 331-338.

36 P. Li, Z. Jin, L. Peng, F. Zhao, D. Xiao, Y. Jin and G. Yu, $A d v$. Mater., 2018, 30, 1-7.

37 Y. Huang, M. Zhong, Y. Huang, M. Zhu, Z. Pei, Z. Wang, Q. Xue, X. Xie and C. Zhi, Nat. Commun., 2015, 6, 10310. 
38 Y. Wang, F. Chen, Z. Liu, Z. Tang, Q. Yang, Y. Zhao, S. Du, Q. Chen and C. Zhi, Angew. Chem., Int. Ed., 2019, 58, 15707-15711.

39 Q. Rong, W. Lei, J. Huang and M. Liu, Adv. Energy Mater., 2018, 8, 1-7.

40 Y. Zhou, C. Wan, Y. Yang, H. Yang, S. Wang, Z. Dai, K. Ji, H. Jiang, X. Chen and Y. Long, Adv. Funct. Mater., 2019, 29, 1806220.

41 L. Wang, G. Gao, Y. Zhou, T. Xu, J. Chen, R. Wang, R. Zhang and J. Fu, ACS Appl. Mater. Interfaces, 2019, 11, 3506-3515.

42 Y. Zhao, Z. Li, S. Song, K. Yang, H. Liu, Z. Yang, J. Wang, B. Yang and Q. Lin, Adv. Funct. Mater., 2019, 29, 1-12.

43 J. Xu, G. Wang, Y. Wu, X. Ren and G. Gao, ACS Appl. Mater. Interfaces, 2019, 11, 25613-25623.

44 F. Lin, Z. Wang, Y. Shen, L. Tang, P. Zhang, Y. Wang, Y. Chen, B. Huang and B. Lu, J. Mater. Chem. A, 2019, 3, 10031-10037.

45 K. Tian, J. Bae, S. E. Bakarich, C. Yang, R. D. Gately, G. M. Spinks, M. In Het Panhuis, Z. Suo and J. J. Vlassak, Adv. Mater., 2017, 29, 1604827.

46 Z. Liu, G. Liang, Y. Zhan, H. Li, Z. Wang, L. Ma, Y. Wang, X. Niu and C. Zhi, Nano Energy, 2019, 58, 732-742.

47 G. Ge, Y. Lu, X. Qu, W. Zhao, Y. Ren, W. Wang, Q. Wang, W. Huang and X. Dong, ACS Nano, 2020, 14, 218-228.

48 S. Lu, J. Shao, K. Ma, D. Chen, X. Wang, L. Zhang, Q. Meng and J. Ma, Carbon N. Y., 2018, 136, 387-394.

49 C. Hu, Y. Zhang, X. Wang, L. Xing, L. Shi and R. Ran, ACS Appl. Mater. Interfaces, 2018, 10, 44000-44010.

50 X. Liu, S. Zou, K. Liu, C. Lv, Z. Wu, Y. Yin, T. Liang and Z. Xie, J. Power Sources, 2018, 384, 214-222.

51 Y. Liang, L. Ye, X. Sun, Q. Lv and H. Liang, ACS Appl. Mater. Interfaces, 2020, 12, 1577-1587.

52 D. Gan, W. Xing, L. Jiang, J. Fang, C. Zhao, F. Ren, L. Fang, K. Wang and X. Lu, Nat. Commun., 2019, 10, 1487.

53 Q. Zhang, X. Liu, L. Duan and G. Gao, Chem. Eng. J., 2019, 365, 10-19.

54 L. Han, K. Liu, M. Wang, K. Wang, L. Fang, H. Chen, J. Zhou and X. Lu, Adv. Funct. Mater., 2018, 28, 1704195.

55 Z. Wang, J. Chen, Y. Cong, H. Zhang, T. Xu, L. Nie and J. Fu, Chem. Mater., 2018, 30, 8062-8069.

56 J. Zhang, L. Wan, Y. Gao, X. Fang, T. Lu, L. Pan and F. Xuan, Adv. Electron. Mater., 2019, 5, 1-10.

57 Z. Wang, H. Zhou, W. Chen, Q. Li, B. Yan, X. Jin, A. Ma, H. Liu and W. Zhao, ACS Appl. Mater. Interfaces, 2018, 10, 14045-14054.
58 J. Chen, Q. Peng, T. Thundat and H. Zeng, Chem. Mater., 2019, 31, 4553-4563.

59 J. Wu, Z. Wu, X. Lu, S. Han, B. R. Yang, X. Gui, K. Tao, J. Miao and C. Liu, ACS Appl. Mater. Interfaces, 2019, 11, 9405-9414.

60 L. M. Zhang, Y. He, S. Cheng, H. Sheng, K. Dai, W. J. Zheng, M. X. Wang, Z. S. Chen, Y. M. Chen and Z. Suo, Small, 2019, 15, 1-8.

61 H. Zhang, W. Niu and S. Zhang, ACS Appl. Mater. Interfaces, 2018, 10, 32640-32648.

62 C. Pang, G. Y. Lee, T. Il Kim, S. M. Kim, H. N. Kim, S. H. Ahn and K. Y. Suh, Nat. Mater., 2012, 11, 795-801.

63 S. Gong, W. Schwalb, Y. Wang, Y. Chen, Y. Tang, J. Si, B. Shirinzadeh and W. Cheng, Nat. Commun., 2014, 5, 3132.

64 H. Wang, B. Zhu, W. Jiang, Y. Yang, W. R. Leow, H. Wang and X. Chen, Adv. Mater., 2014, 26, 3638-3643.

65 X. Peng, H. Liu, Q. Yin, J. Wu, P. Chen, G. Zhang, G. Liu, C. Wu and Y. Xie, Nat. Commun., 2016, 7, 11782.

66 Y. Guo, X. Zhou, Q. Tang, H. Bao, G. Wang and P. Saha, J. Mater. Chem. A, 2016, 4, 8769-8776.

67 Y. Guo, K. Zheng and P. Wan, Small, 2018, 14, 1-9.

68 S. He, L. Qiu, L. Wang, J. Cao, S. Xie, Q. Gao, Z. Zhang, J. Zhang, B. Wang and H. Peng, J. Mater. Chem. A, 2016, 4, 14968-14973.

69 Y. L. Shao, M. F. El-Kady, C. W. Lin, G. Zhu, K. L. Marsh, J. Y. Hwang, Q. Zhang, Y. Li, H. Wang and R. B. Kaner, Adv. Mater., 2016, 28, 6719-6726.

70 L. Gao, Y. Wang, X. Hu, W. Zhou, K. Cao, Y. Wang, W. Wang and Y. Lu, ACS Appl. Mater. Interfaces, 2019, 11, 26288-26297.

71 K. Wang, X. Zhang, C. Li, X. Sun, Q. Meng, Y. Ma and Z. Wei, Adv. Mater., 2015, 27, 7451-7457.

72 W. Li, F. Gao, X. Wang, N. Zhang and M. Ma, Angew. Chem., Int. Ed., 2016, 55, 9196-9201.

73 X. Shi, S. Liu, Y. Sun, J. Liang and Y. Chen, Adv. Funct. Mater., 2018, 28, 1800850.

74 L. Li, Z. Lou, W. Han and G. Shen, Nanoscale, 2016, 8, 14986-14991.

75 Y. Ai, Z. Lou, S. Chen, D. Chen, Z. M. Wang, K. Jiang and G. Shen, Nano Energy, 2017, 35, 121-127.

76 K. Suzuki, K. Yataka, Y. Okumiya, S. Sakakibara, K. Sako, H. Mimura and Y. Inoue, ACS Sens., 2016, 1, 817-825.

77 J. Lee, M. Lim, J. Yoon, M. S. Kim, B. Choi, D. M. Kim, D. H. Kim, I. Park and S. J. Choi, ACS Appl. Mater. Interfaces, 2017, 9, 26279-26285. 